\title{
Robust Stability of Uncertain Systems with Input Delay and Output Feedback Controller
}

\author{
I-Kong Fong and Chii-Ruey Jou \\ Department of Electrical Engineering \\ National Taiwan University \\ Taipei, Taiwan 10617, Republic of China
}

\begin{abstract}
The robust stability of systems with parametric uncertainties, input delays, and output feedback controllers is analyzed in this paper. First, the stability condition of nominal systems is expressed in terms of the eigenvalues of a Hamiltonian matrix. Then, for the nominally stable uncertain system$s$, a new method based on structured singular value and linear fractional transformation techniques is proposed, so that the Lyapunov stability theory can be used to find a set of uncertain paraneters within which the systems remain stable. Finally, it is remarked that the results can be extended to the uncertain systems with multiple time delays.
\end{abstract}

\section{INTRODUCTION}

The robust stability problem of uncertain systems with time delays is usually complicated. Recently, several analysis methods are proposed for such systems with various types of uncertainties $[1,2,3,4,5,6,7]$. However, to consider uncertain time-delay systems with output feedback controller, these methods can not be applied directly, because when there are uncertainties in the input and output matrices of the open-loop system, multiplicative terms of uncertainties will appear in the resulting closedloop system. Especially when there are linearly dependent parametric uncertainties and delays in the state as well as input dynamics, the stability analysis often involves expressions with high order multiplicative terms of the uncertain parameters. Thus straightforward applications of these methods will usually lead to conservative results.

In this paper, the above-mentioned complicated problem is studied. First, with the Lyapunov method, the stability condition of nominal systems is expressed in terms of the eigenvalue locations of a Hamiltonian matrix. Then, for the nominally stable uncertain systems satisfying the condition, a new method based on the structured singular value $[8]$ and linear fractional transformation (LFT) techniques is proposed, so that the Lyapunov stability theory can be used to find a set of uncertain parameters within which the systems remain stable. Finally, it is shown that the results can be extended to the uncertain systems with multiple time delays.

\section{Main Results}

Consider the uncertain single delay system $\left(\Sigma_{s}\right)$ described by the following functional differential equation and controlled by output feedback

$$
\begin{aligned}
\dot{x}(t)= & \left(A_{0}+\sum_{i=1}^{m} k_{i} A_{i}\right) x(t)+\left(D_{0}+\sum_{i=1}^{m} k_{i} D_{i}\right) x(t-h) \\
& +\left(B_{0}+\sum_{i=1}^{m} k_{i} B_{i}\right) u(t-h) \\
y(t)= & \left(C_{0}+\sum_{i=1}^{m} k_{i} C_{i}\right) x(t) \\
x(t)= & \phi_{1}(t) \quad \forall t \in[-h, 0] \\
u(t)= & L y(t),
\end{aligned}
$$

where the state vector $x \in \mathbf{R}^{n}$, the input vector $u \in \mathbf{R}^{p}$, the output vector $y \in \mathbf{R}^{r}$, the output feedback matrix $L \in \mathbf{R}^{p \times r}$, the delay time $h>0$, the initial condition $\phi_{1}$ is a continuous vector function, and the $i$ th uncertain parameter $k_{i} \in \mathbf{R}$ for $i=1, \ldots, m$. It is assumed that $\max _{i}\left|k_{i}\right|<\gamma$, and all $A_{i}, B_{i}, C_{i}, D_{i}$ are known. Note that although it seems that each $k_{i}$ appears in all system matrices, there is no restriction in this formulation of uncertainties, since we can simply set the corresponding $A_{i}$, $B_{i}, C_{i}$, or $D_{i}$ to zero matrices if $k_{i}$ does not affect the particular system matrices. Under these basic assumptions, the closed-loop system is $\dot{x}(t)=\left(A_{0}+\sum_{i=1}^{m} k_{i} A_{i}\right) x(t)+$ $\left(E_{0}+\sum_{i=1}^{m} k_{i} E_{i}+\sum_{i, j=1}^{m} k_{i} k_{j} E_{i j}\right) x(t-h)$, where $E_{0} \triangleq$ $D_{0}+B_{0} L C_{0}, E_{i} \triangleq D_{i}+B_{0} L C_{i}+B_{i} L C_{0}$, and $E_{i j} \triangleq B_{i} L C_{j}$. Let $x_{t}(\sigma) \triangleq x(t+\sigma)$ for $-h \leq \sigma \leq 0$. For the nominal system $\dot{x}(t)=A_{0} x(t)+E_{0} x(t-h)$, we find that a Lyapunov function $V\left(t, x_{t}\right)=x^{T}(t) P x(t)+\int_{t-h}^{t} x^{T}(\sigma) x(\sigma) d \sigma$, where $P$ is some positive definite matrix, can be used to show the stability under some conditions. In fact, we have

Theorem 1: If $A_{0}$ is a stable matrix and for some $\alpha>0$ the Hamiltonian matrix

$$
M=\left[\begin{array}{cc}
A_{0} & E_{0} E_{0}^{T} \\
-(\alpha+1) I_{n} & -A_{0}^{T}
\end{array}\right]
$$

has no eigenvalues on the $j \omega$-axis, then the nominal system is asymptotically stable.

Proof. From the assumptions and Lemma 4 in [9], it is known that the algebraic Riccati equation (ARE)

$$
A_{0}^{T} P+P A_{0}+P E_{0} E_{0}^{T} P+(\alpha+1) I_{n}=0
$$


has a positive definite solution $P$ for some $\alpha>0$. Consider the Lyapunov functional candidate

$$
V\left(t, x_{t}\right)=x^{T}(t) P x(t)+\int_{t-h}^{t} x^{T}(\sigma) x(\sigma) d \sigma .
$$

Then the Lyapunov derivative $\dot{V}\left(t, x_{t}\right)$ is

$$
\begin{aligned}
& x^{T}(t)\left(A_{0}^{T} P+P A_{0}+I_{n}\right) x(t)+2 x(t)^{T} P E_{0} x(t-h) \\
& -x^{T}(t-h) x(t-h) \\
& =\left[\begin{array}{ll}
x^{T}(t) & x^{T}(t-h)
\end{array}\right]\left[\begin{array}{cc}
I_{n} & -P E_{0} \\
0 & I_{n}
\end{array}\right] \text {. } \\
& {\left[\begin{array}{cc}
A_{0}^{T} P+P A_{0}+P E_{0} E_{0}^{T} P+I_{n} & 0 \\
0 & -I_{n}
\end{array}\right] \text {. }} \\
& {\left[\begin{array}{cc}
I_{n} & 0 \\
-E_{0}^{T} P & I_{n}
\end{array}\right]\left[\begin{array}{c}
x(t) \\
x(t-h)
\end{array}\right] \text {. }}
\end{aligned}
$$

Since $P$ is the solution of $\operatorname{ARE~(1),~we~have~} \dot{V}\left(t, x_{t}\right) \leq$ $-\alpha\|x(t)\|^{2}$. Thus, we conclude this theorem by using the Lyapunov stability theorem [10, p. 105].

To proceed, we assume the nominal system satisfies the assumptions of Theorem 1. Thus the nominal system is asymptotically stable, and the ARE (1) has a positive definite solution $P$. We then turn to our second objective: the robust stability of the uncertain system. More specifically, we want to obtain the upper bound $\gamma$ and guarantee the stability of $\left(\Sigma_{s}\right)$ for $\max _{i}\left|k_{i}\right|<\gamma$.

Obviously, we can try to use the same Lyapunov function $V\left(t, x_{t}\right)$ to establish the robust stability. The proof requires one to show that the Lyapunov derivative $\dot{V}\left(t, x_{t}\right)$ is negative definite for all $x_{t}$ and $\max _{i}\left|k_{i}\right|<\gamma$. By some tedious manipulations, it is not difficult to show that $\dot{V}\left(t, x_{t}\right)$ is equal to

$$
\begin{aligned}
& x^{T}(t)\left[P A_{0}+A_{0}^{T} P+I_{n}+\sum_{i=1}^{m} k_{i}\left(P A_{i}+A_{i}^{T} P\right)\right] x(t) \\
& +2 x^{T}(t) P H(k) x(t-h)-x^{T}(t-h) x(t-h) \\
& =\left[\begin{array}{ll}
x^{T}(t) & x^{T}(t-h)
\end{array}\right]\left[\begin{array}{cc}
I_{n} & -P H(k) \\
0 & I_{n}
\end{array}\right] \text {. } \\
& {\left[\begin{array}{cc}
-S(k) & 0 \\
0 & -I_{n}
\end{array}\right]\left[\begin{array}{cc}
I_{n} & 0 \\
-H(k)^{T} P & I_{n}
\end{array}\right]\left[\begin{array}{c}
x(t) \\
x(t-h)
\end{array}\right]}
\end{aligned}
$$

where $k=\left[\begin{array}{lll}k_{1} & \cdots & k_{m}\end{array}\right]^{T}, S(k)=\alpha I_{n}+P E_{0} E_{0}^{T} P-$ $\sum_{i=1}^{m} k_{i}\left(P A_{i}+A_{i}^{T} P\right)-P H(k) H(k)^{T} P$, and $H(k)=E_{0}+$ $\sum_{i=1}^{m} k_{i} E_{i}+\sum_{i, j}^{m} k_{i} k_{j} E_{i j}$. Clearly, $S(k)$ has the form

$$
\begin{aligned}
& Q_{0}-\sum_{i=1}^{m} k_{i} Q_{i}-\sum_{i, j=1}^{m} k_{i} k_{j} Q_{i j} \\
& -\sum_{i, j, \zeta=1}^{m} k_{i} k_{j} k_{\zeta} Q_{i j \zeta}-\sum_{i, j, \zeta, \xi=1}^{m} k_{i} k_{j} k_{\zeta} k_{\xi} Q_{i j \zeta \xi}
\end{aligned}
$$

where $Q_{i}, Q_{i j}, Q_{i j \zeta}, Q_{i j \zeta \xi}$ are all known constant symmetric matrices, and $Q_{0}=\alpha I_{n}$ is nonsingular. Thus, if we want to ensure that $V\left(t, x_{t}\right)$ is negative definite for all $x_{t}$ and $\max _{i}\left|k_{i}\right|<\gamma$, or equivalently, that $S(k)$ is positive definite for all $\max _{i}\left|k_{i}\right|<\gamma$, we only need to ensure the nonsingularity of $S(k)$ for all $\max _{i}\left|k_{i}\right|<\gamma$. The key points are that the eigenvalues of $S(k)$ are continuous functions of $k$, and $S(0)=Q_{0}$ is positive definite. As long as $S(k)$ does not become singular to have zero eigenvalues when $k$ varies, the eigenvalues of $S(k)$ will remain positive. To find the largest value of $\gamma$ such that $S(k)$ is nonsingular for all $\max _{i}\left|k_{i}\right|<\gamma$, we first manage to express $S(k)$ in the form of an LFT. If this can be done, then we have

$$
S(k)=S_{22}+S_{21}\left(I_{\bar{r}}-\Delta S_{11}\right)^{-1} \Delta S_{12},
$$

where the $S_{i j}$ 's are constant matrices which depend only on the $Q$-matrices in (3), $\operatorname{det} S_{22} \neq 0$,

$\Delta=\operatorname{diag}\left\{k_{1} I_{r_{1}}, \ldots, k_{m} I_{r_{m}}\right\}, r_{1}+r_{2}+\cdots+r_{m}=\bar{r}$

and $r_{i}$ 's are positive integers. Under the circumstances, we can use the following theorem [11].

Theorem 2: The LFT of $S(k)$ in (4) is well-defined (i.e., $\left(I_{\bar{r}}-\Delta S_{11}\right)^{-1}$ exists) and $S(k)$ keeps nonsingularity for all $\max _{i}\left|k_{i}\right|<\gamma$ if and only if

$$
\gamma<1 / \max \left\{\mu\left(S_{11}\right), \mu\left(S_{11}-S_{12} S_{22}^{-1} S_{21}\right)\right\},
$$

where $\mu(\cdot)$ is the structured singular value with respect to $\left\{\Delta: k_{i} \in \mathbf{R}\right.$ for $\left.i=1, \ldots, m\right\}$.

Clearly, the $\gamma$ obtained in Theorem 2 serves as an upper bound on $\max _{i}\left|k_{i}\right|$ to keep the asymptotic stability of the uncertain delay system $\left(\Sigma_{s}\right)$. Thus, our development will be complete when we describe how to systematically express $S(k)$ in the form of an LFT in the following. The method we adopted is basically from the works of $[12,13]$, where it is shown how to convert a matrix with mixed unstructured and bounded rational type structured uncertainties to the form of an LFT. However, note our objective here is to use the method to convert a perturbed matrix to the form of an LFT, but not to adjust some uncertain system into the standard configuration suitable for $\mu$-analysis, i.e., a feedback configuration with the nominal systern in the forward path and uncertainty in the return path. Hence there is a fundamental difference between the present work and the previous ones. In view of the fact that we only have structured uncertainties in our perturbed matrix $S(k)$, we only need to describe the part of conversion techniques developed in [13] for that kind of uncertainties.

For our purpose, let $F(M, \Delta)$ denote the LFT

$$
M_{22}+M_{21}\left(I-\Delta M_{11}\right)^{-1} \Delta M_{12},
$$

where $M_{11}, M_{12}, M_{21}$, and $M_{22}$ are constant matrices of suitable dimensions, $M$ is the joint coefficient matrix $\left[\begin{array}{l|l}M_{11} & M_{12} \\ \hline M_{21} & M_{22}\end{array}\right]$, and $\Delta$ has the same diagonal form as the uncertainty matrix $\Delta$ in (5), except the multiplicity of each $k_{i}$ may be different. From (3), it is easy to see that the matrix $S(k)$ has elements that are multivariable polynomials of $k_{i}$ 's. Therefore with the techniques proposed in 
[13], every element of $S(k)$ can be expressed in the form of an LFT $F\left(M^{i j}, \Delta^{i j}(k)\right)$, where $M^{i j}=\left[\begin{array}{l|l}M_{11}^{i j} & M_{12}^{i j} \\ \hline M_{21}^{i j} & M_{22}^{i j}\end{array}\right]$ is a constant joint coefficient matrix, and $\Delta^{i j}(k)$ is a diagonal matrix with elements of $k$ repeated on the diagonal. After transforming the elements of $S(k)$ to LFTs individually, we have

$$
\begin{aligned}
S(k) & =\left[F\left(M^{i j}, \Delta^{i j}(k)\right)\right] \\
& =\bar{M}_{22}+\bar{M}_{21}\left(I_{d}-\bar{\Delta}(k) \bar{M}_{11}\right)^{-1} \bar{\Delta}(k) \bar{M}_{12}
\end{aligned}
$$

where

$$
\begin{aligned}
& \bar{M}_{11}=\operatorname{diag}\left\{M_{11}^{11}, \ldots, M_{11}^{1 n}, \ldots, M_{11}^{n 1}, \ldots, M_{11}^{n n}\right\}, \\
& \bar{M}_{12}=\left[\begin{array}{c}
\operatorname{diag}\left\{M_{12}^{11}, \ldots, M_{12}^{1 n}\right\} \\
\vdots \\
\operatorname{diag}\left\{M_{12}^{n 1}, \ldots, M_{12}^{n n}\right\}
\end{array}\right], \\
& \bar{M}_{21}=\operatorname{diag}\left\{\left[M_{21}^{11} \cdots M_{21}^{1 n}\right], \ldots,\left[M_{21}^{n 1} \cdots M_{21}^{n n}\right]\right\}, \\
& \bar{M}_{22}=\left[\begin{array}{ccc}
M_{22}^{11} & \cdots & M_{22}^{1 n} \\
\vdots & & \vdots \\
M_{22}^{n 1} & \cdots & M_{22}^{n n},
\end{array}\right],
\end{aligned}
$$

$\bar{\Delta}(k)=\operatorname{diag}\left\{\Delta^{11}(k), \ldots, \Delta^{1 n}(k), \ldots, \Delta^{n n}(k)\right\}$, and $I_{d}$ is the identity matrix with the same dimension as that of $\bar{\Delta}(k)$. In general, the size of $\bar{\Delta}(k)$ will be unnecessarily large for $S(k)$ to be expressed as an LFT, and size reduction of $\bar{\Delta}(k)$ is needed. To proceed, $\vec{\Delta}(k)$ can be transformed first by interchanging rows and columns to become a diagonal matrix with elements of $k$ appearing in order, which is exactly the same as the matrix $\Delta$. Note that this rearrangement of the diagonal elements of $\bar{\Delta}(k)$ does not affect the size of the uncertainty matrix, but makes the resulting LFT easier to handle. After this rearrangement, it can be seen that the size reduction of the resulting uncertainty matrix is equivalent to the dimension reduction of a multi-dimensional state-space realization, and the procedure discussed in [13] can be used to reduce the size of the uncertainty matrix. Though the proposed reduction procedure does not guarantee to give a minimal sized uncertainty matrix, examples show that the size reduction is often effectively achieved. No matter the minimal dimension is reached or not, at this stage $S(k)$ has been expressed in the form of (4). Thus our task is completed.

\section{ExTEnsions}

The above procedure can be extended to handle systems with multiple non-commensurate time delays, e.g., system $\left(\Sigma_{m}\right)$ described by

$$
\begin{aligned}
& \dot{x}(t)=\left(A_{0}+\sum_{i=1}^{m} k_{i} A_{i}\right) x(t)+\left(D_{0}+\sum_{i=1}^{m} k_{i} D_{i}\right) x(t-h) \\
& +\sum_{t=1}^{g} \sum_{i=1}^{m} k_{i} F_{i l} x\left(t-h_{l}(t)\right)+\left(B_{0}+\sum_{i=1}^{m} k_{i} B_{i}\right) u(t-h)
\end{aligned}
$$

$$
\begin{aligned}
& y(t)=\left(C_{0}+\sum_{i=1}^{m} k_{i} C_{i}\right) x(t) \\
& x(t)=\phi_{1}(t) \quad \forall t \in\left[-h_{\text {max }}, 0\right] \\
& u(t)=L y(t),
\end{aligned}
$$

where $h_{l}(t), l=1, \ldots, q$, are continuous functions satisfying $0 \leq h_{l}(t) \leq h_{l}<\infty$ and $h_{l}(t) \leq d_{l}<1, h_{\max }=$ $\max _{l} h_{l}$, and $d_{\max }=\max _{l} d_{l}$. The closed-loop system is $\dot{x}(t)=\left(A_{0}+\sum_{i=1}^{m} k_{i} A_{i}\right) x(t)+\sum_{l=1}^{q} \sum_{i=1}^{m} k_{i} F_{i l} x(t-$ $\left.h_{l}(t)\right)+\left(E_{0}+\sum_{i=1}^{m} k_{i} E_{i}+\sum_{i, j=1}^{m} k_{i} k_{j} E_{i j}\right) x(t-h)$, where $E_{0}, E_{i}$, and $E_{i j}$ are defined in Section II. Again, we assume the nominal system with the output feedback gain $L$ is asymptotically stable, but due to the assumption that there exists a positive definite matrix $P$ satisfying the following ARE

$$
A_{0}^{T} P+P A_{0}+P E_{0} E_{0}^{T} P+(\alpha+2) I_{n}=0, \quad \alpha>0 .
$$

This is equivalent to assuming that the ARE in (1) has a positive definite solution for some $\alpha>1$. In other words, we make a little stronger assumption to cope with the additional delay terms in $\left(\Sigma_{m}\right)$.

For the uncertain system $\left(\Sigma_{m}\right)$, consider the Lyapunov functional candidate $V\left(t, x_{t}\right)=$

$$
x^{T}(t) \operatorname{Px}(t)+\int_{t-h}^{t} x^{T}(\sigma) x(\sigma) d \sigma+\frac{1}{q} \sum_{l=1}^{q} \int_{t-h_{l}(t)}^{t} x^{T}(\sigma) x(\sigma) d \sigma .
$$

Then Lyapunov derivative $\dot{V}\left(t, x_{t}\right)$ can be written as

$$
\begin{aligned}
& x^{T}(t)\left[P A_{0}+A_{0}^{T} P+2 I_{n}+\sum_{i=1}^{m} k_{i}\left(P A_{i}+A_{i}^{T} P\right)\right] x(t) \\
& +2 x^{T}(t) P H(k) x(t-h)-x^{T}(t-h) x(t-h) \\
& +2 x^{T}(t) P \sum_{i=1}^{q} \sum_{i=1}^{m} k_{i} F_{i l} x\left(t-h_{l}(t)\right) \\
& -\frac{1}{q} \sum_{i=1}^{q}\left(1-\dot{h}_{l}(t)\right) x^{T}\left(t-h_{l}(t)\right) x\left(t-h_{l}(t)\right) \\
& \leq \boldsymbol{x}^{T}(t) \boldsymbol{T} Q T^{T} x(t)
\end{aligned}
$$

where $x(t)=\left[x^{T}(t) x^{T}(t-h) x^{T}\left(t-h_{1}(t)\right) \cdots x^{T}(t-\right.$ $\left.\left.h_{q}(t)\right)\right]^{T}, T$ is an upper triangular matrix with unities along its diagonal, $Q=-\operatorname{diag}\left\{S_{m}(k), I_{n}, \frac{1-d_{\max }}{q} I_{q n}\right\}$, and $S_{m}(k)=\alpha I_{n}-\sum_{i=1}^{m} k_{i}\left(P A_{i}+A_{i}^{T} P\right)-P H(k) H(k)^{T} P$ $-\frac{q}{2\left(1-d_{\max }\right)} \sum_{i, j=1}^{m} k_{i} k_{j} \sum_{l=1}^{q} P\left(F_{i l} F_{j l}^{T}+F_{j l} F_{i l}^{T}\right) P+$ $P E_{0} E_{0}^{T} P$. Thus the remaining task is to ensure that $S_{m}(k)$ is positive definite, and the rest of the discussions follow the same line of development as in Section II, except now the LFT of $S_{m}(k)$ is in general different from that of $S(k)$.

Moreover, it is also possible to use the same approach on the even more general system. For example, the system $\left(\Sigma_{m}\right)$ with input part changed to $\left(B_{0}+\sum_{i=1}^{m} k_{i} B_{i}\right) u(t)+$ $\left(\tilde{B}_{0}+\sum_{i=1}^{m} k_{i} \tilde{B}_{i}\right) u(t-h)$ and/or output part changed to 
$\left(C_{0}+\sum_{i=1}^{m} k_{i} C_{i}\right) x(t)+\left(\tilde{C}_{0}+\sum_{i=1}^{m} k_{i} \tilde{C}_{i}\right) x(t-h)$ can be handled similarly. The only difference is that the corresponding Lyapunov derivative will be more complicated.

\section{AN EXAMPLE}

Consider an uncertain system $\left(\Sigma_{s}\right)$ with the following data: $A_{0}=\left[\begin{array}{rr}-9 & 2 \\ -1 & -8\end{array}\right], \quad B_{0}=\left[\begin{array}{l}1 \\ 1\end{array}\right], \quad C_{0}^{T}=$ $\left[\begin{array}{r}0.5 \\ 1\end{array}\right], \quad D_{0}=\left[\begin{array}{ll}0.5 & 0 \\ 0.75 & 1\end{array}\right], A_{1}=\left[\begin{array}{ll}0 & 1 \\ 1 & 0\end{array}\right], \quad B_{1}=$ $\left[\begin{array}{l}0 \\ 1\end{array}\right], C_{2}^{T}=\left[\begin{array}{l}0 \\ 1\end{array}\right], D_{1}=\left[\begin{array}{ll}0 & 0 \\ 0 & 1\end{array}\right], A_{2}, B_{2}, C_{1}$, and $\mathrm{D}_{2}$ are zero matrices of suitable dimensions, and the output feedback gain $L=-0.5$. By using Theorem 1 , it is found that ARE (1) has a positive definite solution

$$
P=\left[\begin{array}{ll}
52.08 & 2.640 \\
2.640 & 16.38
\end{array}\right]
$$

at $\alpha=125$. Hence the nominal system is asymptotically stable. From these information, $S(k)=\left[\begin{array}{ll}s_{11} & s_{12} \\ s_{21} & s_{22}\end{array}\right]$ can be obtained, where $s_{12}=s_{12}$, and $s_{11}, s_{12}$ and $s_{22}$ are, respectively,

$$
\begin{aligned}
& 125+78.91 k_{1}-1353 k_{2}-2.178 k_{1}^{2}-748.6 k_{2}^{2} \\
& +6.969 k_{1} k_{2}+3.484 k_{1}^{2} k_{2}-72.23 k_{1} k_{2}^{2}-1.742 k_{1}^{2} k_{2}^{2}, \\
& 189.5 k_{1}-47.13 k_{2}-13.51 k_{1}^{2}-260.2 k_{2}^{2} \\
& +43.23 k_{1} k_{2}+21.62 k_{1}^{2} k_{2}-236.6 k_{1} k_{2}^{2}-10.81 k_{1}^{2} k_{2}^{2}, \\
& 125-45.31 k_{1}+130.6 k_{2}-83.82 k_{1}^{2}-90.41 k_{2}^{2} \\
& +268.2 k_{1} k_{2}+134.1 k_{1}^{2} k_{2}-155.7 k_{1} k_{2}^{2}-67.05 k_{1}^{2} k_{2}^{2} .
\end{aligned}
$$

Following the procedure described in Section II and using the $\mu$-analysis package [14], it is possible to get a bound of 0.08 on $\max _{i}\left|k_{i}\right|$ to keep the nonsingularity of $S(k)$, and hence the asymptotic stability of the above system.

\section{Conclusion}

In this paper we develop an LFT approach to deal with the robust stability analysis problem of delay systems. A condition on the stability of the nominal systern is proposed in terms of the eigenvalue locations of a Hamiltonian matrix. Then a systematic procedure is described for the computations of the bound of the uncertain parameters to keep the stability. Note that because the Lyapunov theory is used, the stability is guaranteed even for time-varying uncertainties. As the main objective of this paper is to introduce a new method for transforming the robust stability problem into a manageable form, the numerical cornputation issues related to the structured singular values are not addressed. These issues, including how to find an acceptable bound with simple computation requirements, will be discussed in the future papers.

\section{ACKNOWLEDGMENT}

This research is supported by the National Science Council of the Republic of China under Grant NSC 830404-E002-004.

\section{REFERENCES}

[1] S. S. Wang, B. S. Chen, and T. P. Lin, "Robust stability of uncertain time-delay systems," Int. J. Control, vol. 46, pp. 963-976, 1987.

[2] J. H. Chou, I. R. Horng, and B. S. Chen, "Dynamical feedback compensator for uncertain time-delay systems containing saturating actuator," Int. J. Control, vol.49, pp. 961-968, 1989.

[3] E. Cheres, Z. J. Palmor, and S. Gutman, "Quantitative measures of robustness for systems including delayed perturbations," IEEE Trans. Automat. Control, vol. 34, pp. 1203-1204, 1989.

[4] A. Hmamed, "Further results on the robust stability of uncertain time-delay systems," Int. J. Systems Sci., vol. 22, pp. 605-614, 1991.

[5] S. Phoojaruenchanachai and K. Furuta, "Memoryless stabilization of uncertain linear systems including time-varying state delays," IEEE Trans. Automat. Control, vol. 37, pp. 1022-1026, 1992.

[6] T. J. Su and C. G. Huang, "Robust stability of delay dependence for linear uncertain systems," IEEE Trans. Automat. Control, vol. 37, pp. 1656-1659, 1992.

[7] W. Yu, K. M. Sobel, and E. Y. Shaprio, "A time domain approach to the robustness of time delay systems," Proc. of the 31st IEEE Conf. on Decision and Control, Tucson, Arizona, pp. 3726-3727, 1992.

[8] J. C. Doyle, "Analysis of feedback systems with structured uncertainties," Proc. IEE, pt. D, vol. 129, pp. $242-250,1982$.

[9] J. C. Doyle, K. Glover, P. P. Khargoneckar, and B. A. Francis, "State-space solutions to standard $\mathrm{H}_{2}$ and $H_{\infty}$ control problem," IEEE Trans. Automat. Control, vol. 34, pp. 831-846, 1989.

[10] J. Hale, Theory of Functional Differential Equations, New York, NY: Springer-Verlag, 1977.

[11] A. K. Packard, What's New With $\mu$ : Structured Uncertainty in Multivariable Control. Ph. D. dissertation, University of California, Berkeley, 1988.

[12] M. Steinbuch, J. C. Terlouw, O. H. Bosgra, and S. G. Smit, "Uncertainty Modeling and Structured Singular Value Computation Applied to an Electromechanical System," Proc. IEE, pt. D, vol. 139, pp. 301-307, 1992.

[13] P. Lambrechts, J. Terlouw, S. Bennani, and M. Steinbuch, "Parametric uncertainty modeling using LFTs," Proc. American Contr. Conf., San Francisco, California, pp. 267-272, 1993.

[14] G. J. Balas, J. C. Doyle, K. Glover, A. Packard, and $R$. Smith, $\mu$-Analysis and Synthesis Toolbox-User's Guide, Natick, MA: Musyn Inc. and The MathWorks Inc., 1991 . 